УДК 534.21

\title{
МАТЕМАТИЧЕСКОЕ МОДЕЛИРОВАНИЕ ПРОЦЕССА УЛЬТРАЗВУКОВОЙ ПОДГОТОВКИ ВЫСОКОВЯЗКОЙ НЕФТИ К ТРАНСПОРТУ
}

\author{
Азин Антон Владимирович1, \\ antonazin@niipmm.tsu.ru
}

\author{
Богданов Евгений Петрович, \\ epbogdanov@mail.ru
}

\author{
Марицкий Николай Николаевич ${ }^{1}$ \\ nnmar@niipmm.tsu.ru
}

\section{Пономарев Сергей Александрович1, doc.sergeyponomarev@gmail.com}

\author{
Рикконен Сергей Владимирович 1 , \\ rikk2@yandex.ru \\ 1 Национальный исследовательский Томский государственный университет, \\ Россия, 634050, г. Томск, пр. Ленина, 36. \\ 2 Национальный исследовательский Томский политехнический университет, \\ Россия, 634050, г. Томск, пр. Ленина, 30.
}

\begin{abstract}
Актуальность исследования обусловлена необходимостью определения уровня энергии физических воздействий на высоковязкие нефрти с целью их подготовки к транспорту. Эти знания актуальны для проектирования ультразвуковых установок по изменению реологических свойств нефти.

Цель: определение уровней акустической энергии в слоях многослойной акустической системы при разных частотах воздействия для выявления резонансных режимов работы механической системы «излучатель-нефрть» с целью подготовки нефрти к транспорту.

Объекты: механическая многослойная система «излучатель-нефть», конструкция которой определяется способом внесения энергии и резонансной частотой конкретного слоя системы.

Методы: математическое моделирование процесса ультразвукового излучения с определением энергии прохождения, поглощения и отражения в каждом слое механической системы «излучатель-нефрть», определение резонансных частот с максимальным уровнем энергии в неффтяном слое.

Результаты. Разработана математическая модель многослойной акустической системы, позволяющая рассчитать энергию акустического излучения в каждом слой системы. Частотные характеристики акустического излучения позволяют определить резонансные режимы в каждом слое системы, в том числе в нефти. Знания уровня акустической энергии в последующем позволит определить изменения реологических свойств нефти, в том числе и от нагрева.

Выводы. Математическая модель распространения ультразвука в многослойной среде позволяет рассчитать энергию в каждом слое акустической системы при различных частотах возбуждения источника колебаний с различными соотношениями длины волны в слое и толщины данного слоя. Наличие отраженных волн в каждом слое создает череду резонансов (режимов стоячих волн). Частотные характеристики с учетом интерфреренционной картины поля отражают картину проникновения энергии в слои и показывают, что за счет образования стоячих волн (резонансов) энергия в нефрти может быть близка к энергии излучателя. Акустическая энергия в слое нефти падает в зависимости от увеличения исходной вязкости. Данная математическая модель позволяет рассчитать конструкцию акустической системы для подготовки нефти и углеводородных топлив к транспорту, подготовки топлив к сжиганию, подготовки масел к эксплуатации в условиях Арктики и Антарктики.
\end{abstract}

\section{Ключевые слова:}

Ультразвуковое излучение, энергия излучения, стоячие волны, отраженные волны, частотные характеристики, слоистая акустическая система.

Наличие надмолекулярной структуры высоковязких нефтей за счет повышения вязкости вызывает ряд проблем, возникающих в процессе добычи, перекачки и переработки данного сырья. Для перечисленных технологических процессов это выражается в высокой (положительной) температуре застывания и отложениях парафина на оборудовании и трубопроводе. В настоящее время применяют ряд методов для подготовки высоковязкой нефти к транспорту: термиче- ский, химический и метод физических воздействий. На сегодняшний день физические методы находят все более широкое применение в нефтяной промышленности из-за их эффективности, экономичности и доступности [1-5]. Одним из физических методов является метод ультразвукового (УЗ) воздействия. Теме воздействия ультразвука на углеводородные среды посвящено достаточно много научно-технических работ [5-16]. В этих работах показано влияние УЗ на 
реологические свойства углеводородной среды. Эффект воздействия ультразвука связан с уровнем акустической энергии, переданной в среду. Для проектирования конкретных устройств, предназначенных для подготовки нефти и углеводородных топлив к транспорту, подготовки топлив к сжиганию, подготовки масел к эксплуатации в условиях Арктики и Антарктики, требуется знать уровни акустической энергии во всех элементах рассматриваемой конструкции. Математическое моделирование и расчет акустической энергии, сообщенной в многослойную среду, являются актуальными задачами.

Целью работы является построение математической модели проникновения УЗ излучения через многослойную систему для нагрева высоковязкой углеводородной среды.

Расчет энергии излучения, концентрированной в слое нефти, позволит в дальнейшем определиться с такими физическими процессами в среде, как: скорость акустического течения; акустическая кавитация и нагрев нефти с изменением реологических свойств нефти [2-5].

Рассмотрено распространение плоской волны в многослойной (пятислойной) акустической системе, представленной на рис. 1. Характерное свойство плоской акустической бегущей волны - это перенос энергии без переноса вещества. К понятию плоской волны применено понятие одномерной волны. Одномерные волны - это волны, в которых все характеристики зависят, помимо времени, только от одной координаты.

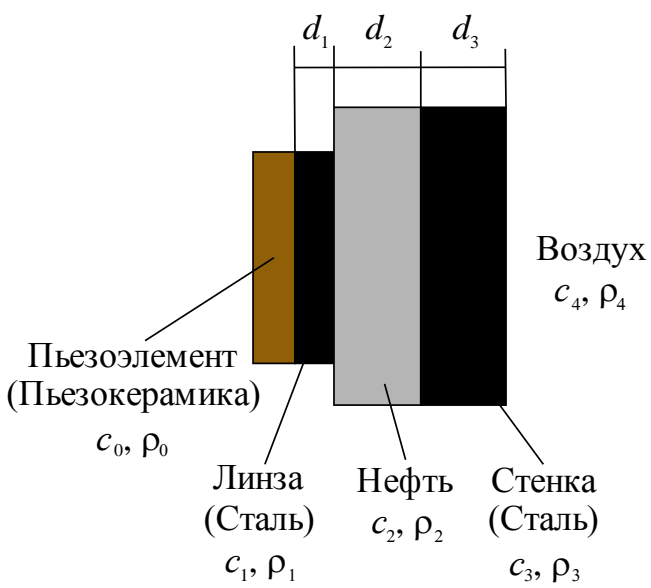

Pис. 1. Схема пятислойной системы: $d_{1}-$ толщиина стальной линзы; $d_{2}-$ толщина нефтяного слоя; $d_{3}-$ толщина стальной стационарной стенки; $c_{0}$ $c_{1}, c_{2}, c_{3}, c_{4}-$ скорость звука в материалах; $\rho_{0}, \rho_{1}$, $\rho_{2}, \rho_{3}, \rho_{4}-$ плотность материалов

Fig. 1. Model of a five-layer system: $d_{1}-$ steel lens thickness; $d_{2}-$ oil layer thickness; $d_{3}-$ stationary steel wall thickness; $c_{0}, c_{1}, c_{2}, c_{3}, c_{4}$-speed of sound in materials; $\rho_{0}, \rho_{1}, \rho_{2}, \rho_{3}, \rho_{4}$-density of materials

В бегущей плоской волне модуль вектора мгновенного значения потока мощности $W_{i}$ равен [17-20]:

$$
W_{i}=P V=\frac{1}{\rho c} P^{2}=\rho c V^{2}=\rho c \omega^{2} A_{i}^{2} \mathrm{BT} / \mathrm{M}^{2},
$$

где $P$ - давление (Па), $V$ - скорость среды (м/с), $\rho-$ плотность среды (кг/M $\left.{ }^{3}\right), c-$ фазовая скорость волны $(\mathrm{M} / \mathrm{c}), \omega-$ угловая частота $(1 / \mathrm{c}), A_{i}$ - мгновенная амплитуда колебаний среды (м).

Модуль вектора мгновенного значения потока мощности $W_{i}$ подразумевает наличие фазы вектора в пространстве (1). Для плоской продольной волны распространение потока энергии происходит по одной оси пространства, и фаза потока означает, в том числе, расхождение максимума давления и максимума колебательной скорости вдоль распространения потока акустической энергии. Для определения плотности потока энергии волны используется вектор Умова, направленный в сторону распространения волны (т. е. перпендикулярно волновой поверхности), величина которого равна произведению объемной плотности энергии и скорости распространения волны. Для энергетической характеристики звукового поля плоской волны вводится понятие «интенсивность звука» [18-20].

Интенсивность волны [6-9] равна среднему значению модуля вектора Умова:

$$
\bar{I}=\langle P\rangle=\left\langle W_{i}\right\rangle c,
$$

где $\langle P\rangle$ - среднее значение модуля вектора Умова; $\left\langle W_{i}\right\rangle$ - среднее значение мгновенной полной звуковой мощности; $c$ - фазовая скорость.

Для плоской бегущей гармонической волны средняя интенсивность, согласно (2), равна (3) [18-20]:

$$
\bar{I}=\frac{1}{2} c \rho \omega^{2} A^{2} \mathrm{BT} / \mathrm{M}^{2} .
$$

Для проектирования ультразвуковых систем необходимо учитывать волновую картину процесса передачи энергии, зависимую от соотношения длины волны и толщины слоя сплошной среды, в которой распространяется звуковая энергия.

При учете волновой картины звукового поля в слое необходимо учитывать возможность появления режима стоячих волн, при котором интенсивность звука слоя может в несколько раз превышать интенсивность источника звука [18-20].

Важным моментом является поглощение энергии в среде, которое существенно изменяет интерференционную картину процесса передачи энергии. При близких значениях длины волны и толщины слоя материала волновая (интерференционная) картина усложняется. Учет интерференционных процессов и процесса поглощения в многослойной акустической системе вызывает существенные трудности при расчете режимов передачи энергии от источника энергии в конкретный слой многослойной системы. Для правильного понимания полной картины звукового поля и учета соотношения длины волны и толщины слоя необходимо использовать вектор Умова в следующем виде:

$$
\begin{gathered}
p=p_{0} e^{i(\omega t-k x)}, \\
\dot{X}=\dot{X}_{0} e^{i(\omega t-k x+\varphi)} .
\end{gathered}
$$




$$
\begin{gathered}
P_{i}=p_{0} e^{i(\omega t-k x)} \dot{X}_{0} e^{i(\omega t-k x+\varphi)}= \\
=\frac{p_{0}^{2}}{\rho_{0} c_{0}} e^{i(\omega t-k x)} e^{i(\omega t-k x+\varphi)}= \\
=\rho_{0} c_{0} \dot{X}_{0}^{2} e^{i(\omega t-k x)} e^{i(\omega t-k x+\varphi)}= \\
=I_{0} e^{i(\omega t-k x)} e^{i(\omega t-k x+\varphi)} .
\end{gathered}
$$

Принимая во внимание (4), получено уравнение $(5)$, где $p_{0}$ и $\dot{X}_{0}-$ акустическое давление и виброскорость источника излучения; $P_{i}-$ мгновенное значение вектора Умова в слое, $I_{0}=\dot{X}_{0}^{2} p_{0} c_{0}-$ амплитуда вектора Умова источника излучения; $k=\omega \sqrt{\frac{\rho}{E}}=\frac{\omega}{c}=\frac{2 \pi}{\omega}-$ волновое число; $x-$ толщина слоя. Фазовая скорость $c=\frac{\omega}{k}=\sqrt{\frac{E}{\rho}}$, где $E-$ модуль Юнга; $T=\frac{1}{f}=\frac{2 \pi}{\omega}-$ период колебания.

В разработанной математической модели процесса ультразвуковой подготовки высоковязкой нефти к транспорту за счет нагрева приняты допущения:

- рассматривается только акустическая энергия плоской волны в каждом слое конструкции и, в частности, в нефти;

- скорость акустического течения принимается равной нулю. Она зависит от вязкости среды, интенсивности ультразвука и его частоты. Скорость течения мала и составляет доли процента от скорости ультразвука;

- не учитывается акустическая кавитация;

- температура нефти постоянна;

- нефть представляется как углеводородная среда с ньютоновскими свойствами (динамическая вязкость и плотность среды постоянны).

В математической модели учитывается динамическая вязкость, выраженная в (Па·с). Коэффициент поглощения акустической энергии материала определяется по [4]:

$$
\alpha 2=\frac{2 \mu_{\mathrm{H}} \omega^{2}}{3 \rho c^{3}} 1 / \mathrm{M} .
$$

Коэффициент поглощения нефти $\alpha 2$ при динамической вязкости $\mu_{\mathrm{H}}=0,5$ Па с рассчитан по формуле (6) [1-3]:

$$
\alpha 2=0,149 \cdot 10^{-10} \cdot \omega^{2} \text { Дб/м. }
$$

Коэффициенты поглощения керамики, стали и воздуха определяются экспериментальным путем и зависят от изменения угловой частоты [20]:

- керамика: $\alpha 0=5,1 \cdot 10^{-14} \cdot \omega^{2}$, Дб/м;

- сталь: $\alpha 1=5,5 \cdot 10^{-14} \cdot \omega^{2}$, Дб/м;

- сталь: $\alpha 3=5,5 \cdot 10^{-14} \cdot \omega^{2}$, Дб/м;

- воздух: $\alpha 4=15,9 \cdot 10^{-6} \cdot \omega^{2}$, Дб/м.

Система уравнений пятислойной акустической колебательной системы с учетом отраженных волн, поглощения материалом акустической энергии, с учетом волновой (интерференционной) картины в слоях:

$$
\begin{aligned}
& P_{0}=I_{j} e^{-2 \alpha 0_{(i+1)} d_{0}} e^{i 2\left(\frac{\omega_{(i+1)} t_{(i+1)}}{N_{(i+1)}}-k 0_{(i+1)} d_{0}\right)}- \\
& -R_{0}^{2} I_{j} e^{-4 \alpha 0_{(i+1)} d_{0}} e^{i 2\left(\frac{\omega_{(i+1)} t_{(i+1)}}{N_{(i+1)}}+k 0_{(i+1)} d_{0}\right)} ; \\
& P_{1}=P_{0} e^{-2 \alpha 1_{(i+1)} d_{1}} e^{i 2\left(\frac{\omega_{(i+1)} t_{(i+1)}}{N_{(i+1)}}-k 1_{(i+1)} d_{1}\right)}- \\
& -R_{1}^{2} P_{0} e^{-4 \alpha 0_{(i+1)} d_{1}} e^{i 2\left(\frac{\omega_{(i+1)} t_{(i+1)}}{N_{(i+1)}}+k 1_{(i+1)} d_{1}\right)} ; \\
& P_{2}=P_{1} e^{-2 \alpha 2_{(i+1)} d_{2}} e^{i 2\left(\frac{\omega_{(i+1)} t_{(i+1)}}{N_{(i+1)}}-k 2_{(i+1)} d_{2}\right)}- \\
& -R_{2}^{2} P_{1} e^{-4 \alpha 2_{(i+1)} d_{2}} e^{i 2\left(\frac{\omega_{(i+1)} t_{(i+1)}}{N_{(i+1)}}+k 2_{(i+1)} d_{2}\right)} ; \\
& P_{3}=P_{2} e^{-2 \alpha 3_{(i+1)} d_{3}} e^{i 2\left(\frac{\omega_{(i+1)} t_{(i+1)}}{N_{(i+1)}}-k 3_{(i+1)} d_{3}\right)}- \\
& -R_{3}^{2} P_{2} e^{-4 \alpha 3_{(i+1)} d_{3}} e^{i 2\left(\frac{\omega_{(i+1)} t_{(i+1)}}{N_{(i+1)}}+k 3_{(i+1)} d_{3}\right)} ; \\
& P_{4}=P_{3} e^{-2 \alpha 4_{(i+1)} d_{4}} e^{i 2\left(\frac{\omega_{(i+1)} t_{(i+1)}}{N_{(i+1)}}-k 4_{(i+1)} d_{4}\right)} d_{4} e^{i 2\left(\frac{\omega_{(i+1)} t_{(i+1)}}{N_{(i+1)}}+k 4_{(i+1)} d_{4}\right)}
\end{aligned}
$$

где $P_{0}-$ вектор Умова в пьезокерамическом слое, Вт/м² $P_{1}$ - вектор Умова в слое стали; $P_{2}-$ вектор Умова в слое нефти; $P_{3}$ - вектор Умова в слое стали; $P_{4}$ - вектор Умова в воздушном слое, Вт/м²; коэффициенты отражения для слоев: $R_{0}=\frac{\rho_{1} c_{1}-\rho_{0} c_{0}}{\rho_{1} c_{1}+\rho_{0} c_{0}}$, $R_{1}=\frac{\rho_{2} c_{2}-\rho_{1} c_{1}}{\rho_{2} c_{2}+\rho_{1} c_{1}}, R_{2}=\frac{\rho_{3} c_{3}-\rho_{2} c_{2}}{\rho_{3} c_{3}+\rho_{2} c_{2}}, R_{3}=\frac{\rho_{4} c_{4}-\rho_{3} c_{3}}{\rho_{4} c_{4}+\rho_{3} c_{3}}, R_{4}=0$; $\alpha_{i}$ - коэффициенты поглощения слоев акустического поля, дБ/м; $k 0_{(i+1)}=\frac{\omega_{(i+1)}}{c_{0}}, k 1_{(i+1)}=\frac{\omega_{(i+1)}}{c_{1}}, k 2_{(i+1)}=\frac{\omega_{(i+1)}}{c_{2}}$, $k 3_{(i+1)}=\frac{\omega_{(i+1)}}{c_{3}}, k 4_{(i+1)}=\frac{\omega_{(i+1)}}{c_{4}}-$ волновые числа слоев, $1 / \mathrm{M} ; I_{j}=\frac{1}{2} \dot{X}^{2} \rho_{0} c_{0}-$ интенсивность акустического излучения источника, Вт/м² $;$ - толщина слоев акустической системы, м. Здесь время $t$ учитывается за 1 секунду (все функции гармонические и периодические), плюс часть периода поворота угла $\omega t$ на долю периода $\Delta$. Принимается:

$$
t_{(i+1)}=1+\frac{\mathrm{T}_{(i+1)}}{\Delta},
$$

где TП $_{(i+1)}=\frac{1}{f_{(i+1)}}-$ текущий период колебания процесса, $\Delta$ - доля периода $(1 / 4,1 / 2,3 / 4,1)$. Значение фазы круговой частоты на времени 1 секунда будет равно $\frac{\omega_{(i+1)} t}{N_{(i+1)}}$, где $N_{(i+1)}-$ количество колебаний в 1 секунду (численно равно частоте колебаний). Площадь акустической системы принимается равной $1 \mathrm{~m}^{2}$. 

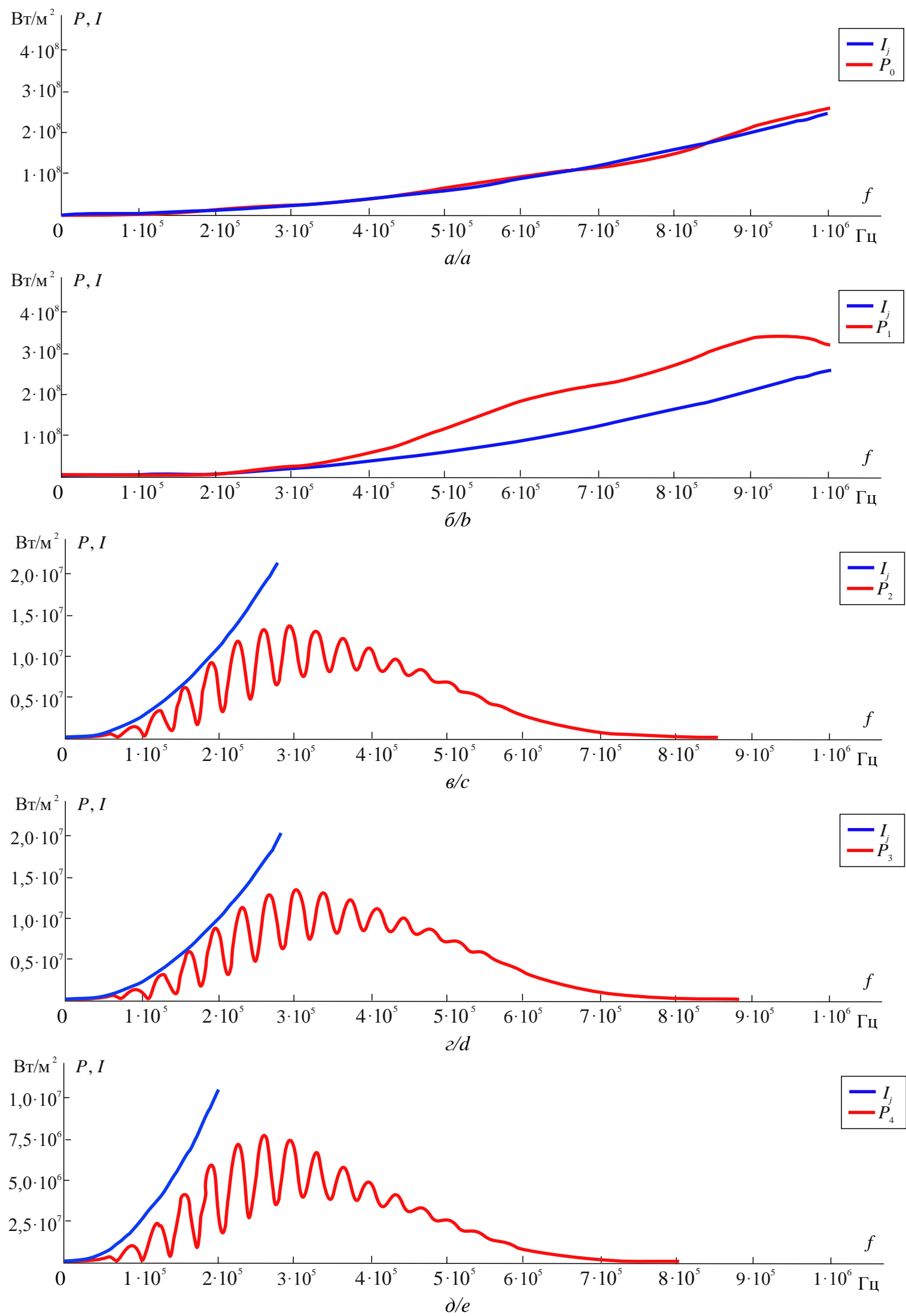

Рис. 2. Частотная характеристика интенсивностей звуковой энергии в слоях акустической системьл: а) пьезокерамика, $d_{0}=2 \cdot 10^{-3} \mathrm{M}$; б) сталь, $d_{1}=1 \cdot 10^{-3}{ }_{\mathcal{M}}$; в) нефть, $d_{2}=1 \cdot 10^{-2} \mathrm{M}$; г) сталь, $\left.d_{3}=1 \cdot 10^{-3}{ }_{\mathcal{M}} ; \partial\right)$ воздух, $d_{4}=1 \cdot 10^{-2} \mathrm{M}$

Fig. 2. Frequency characteristic of sound energy intensity in the acoustic system layers: a) piezoelectric ceramics, $d_{0}=1 \cdot 10^{-2} m$; b) steel, $d_{1}=1 \cdot 10^{-3} \mathrm{~m} ; \mathrm{c}$ ) oil, $d_{2}=1 \cdot 10^{-2} \mathrm{~m} ; \mathrm{d}$ ) steel, $d_{3}=1 \cdot 10^{-3} \mathrm{~m} ; \mathrm{e}$ ) air, $d_{4}=1 \cdot 10^{-2} \mathrm{~m}$ 
Решение системы уравнений (7) проводилось численным методом в пакете прикладных программ Mathcad. На pис. 2 представлены частотные характеристики векторов Умова в слоях акустической системы, которые включают пьезокерамику излучателя, стальную линзу, слой нефти, стальную стенку, воздушное пространство.

По частотным характеристикам интенсивностей в слоях заметно, что режим стоячих волн начинает проявляться в стальном слое линзы излучателя (рис. 2). В слое нефти, корпуса из стали и воздушного пространства режимы стоячих волн реализуются в виде резонансных пиков и антирезонансных провалов.

На волновую картину процесса передачи энергии будет влиять соотношение $\lambda$ длины волны в слое

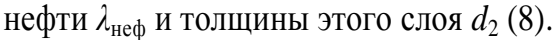

$$
\lambda=\frac{\lambda_{\text {неф }}}{4}-d_{2}=0 .
$$

На рис. 3 приведены результаты моделирования. Если толщина слоя составляет небольшую долю от длины волны $\frac{\lambda_{\text {неф }}}{4}>d_{2}$, заметного отражения звуковой волны не будет, звуковая волна пройдет препятствие, как если бы его не было [17].

Начиная с частоты воздействия, при которой $\frac{\lambda_{\text {неф }}}{4}=d_{\text {слоя }}$, появляются режимы стоячих волн, проявляющиеся в виде резонансных пиков и антирезонансных провалов (рис. 3).

При дальнейшем возрастании частоты воздействия резонансные явления пропадают, при $\frac{\lambda}{4}<d_{\text {слоя }}$ толщина слоя велика по сравнению с длиной волны. В этом случае за слоем образуется звуковая тень. Только на частотах 500 кГц режимы стоячих волн перестают оказывать влияние на передачу энергии (рис. 3).

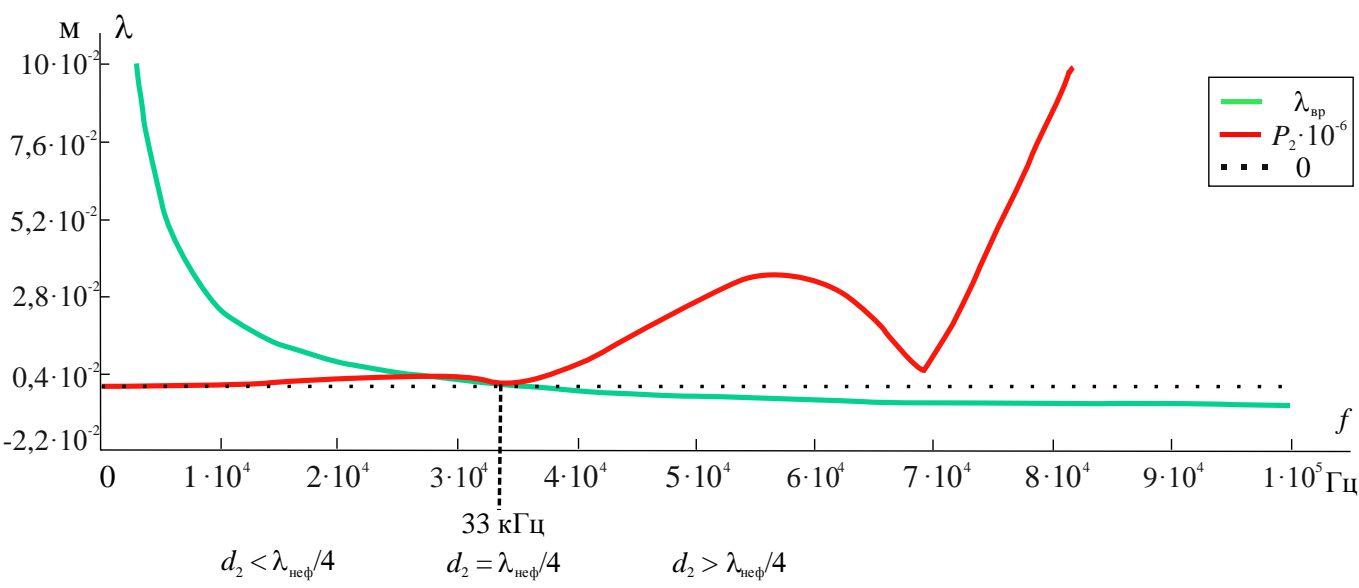

Puc. 3. Соотношение длины волны $\lambda_{\text {неф }}$ и толщины нефтяного слоя $d_{2}$

Fig. 3. Ratio of wavelength $\lambda_{\text {oil }}$ and thickness of the oil layer $d_{2}$

Для оценки энергии звука в нефти введено отношение энергии звука в нефти к энергии звука источника УЗ излучения:

$$
\eta_{\mathrm{H}}=\frac{P_{\mathrm{H}}}{P_{j}}
$$

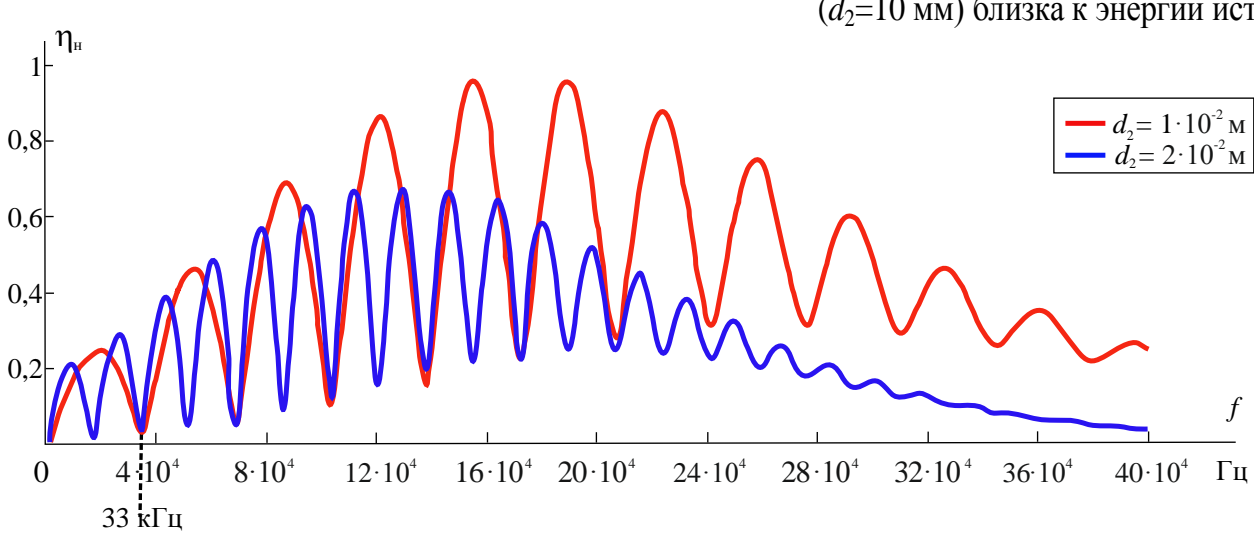

Рис. 4. Частотная зависимость коэффициента интенсивности $\eta_{н}$ при толщине слоя нефти $d_{2}=1 \cdot 10^{-2} \mathrm{M} и d_{2}=2 \cdot 10^{-2} \mathrm{M}$, $\mu_{H}=0,5 \Pi a \cdot c$

Fig. 4. Frequency dependence of intensity coefficient $\eta_{n}$ with oil thickness $d_{2}=1 \cdot 10^{-2} \mathrm{~m}$ and $d_{2}=2 \cdot 10^{-2} \mathrm{~mm}, \mu_{n}=0,5 \mathrm{~Pa} \cdot \mathrm{s}$ где $\eta_{\text {н }}$ - отношение энергии звукового поля в конкрет-

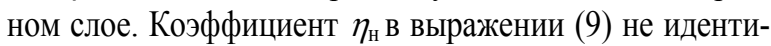
чен коэффициенту пропускания и отражает появление резонансных явлений в слоях акустической системы. В случае со слоем углеводородной природы интенсивность звука в слое нефти на некоторых частотах $\left(d_{2}=10\right.$ мм) близка к энергии источника (рис. 4$)$. 
Частотные характеристики с учетом интерференционной картины поля отражают картину проникновения энергии в слои и показывают, что за счет образования стоячих волн и накопления энергии в слое отношение $\eta_{н}$ данного слоя к входящей энергии (при малых поглощениях в слоях) может быть близко к 1 или даже превышать 1 (рис. 5).
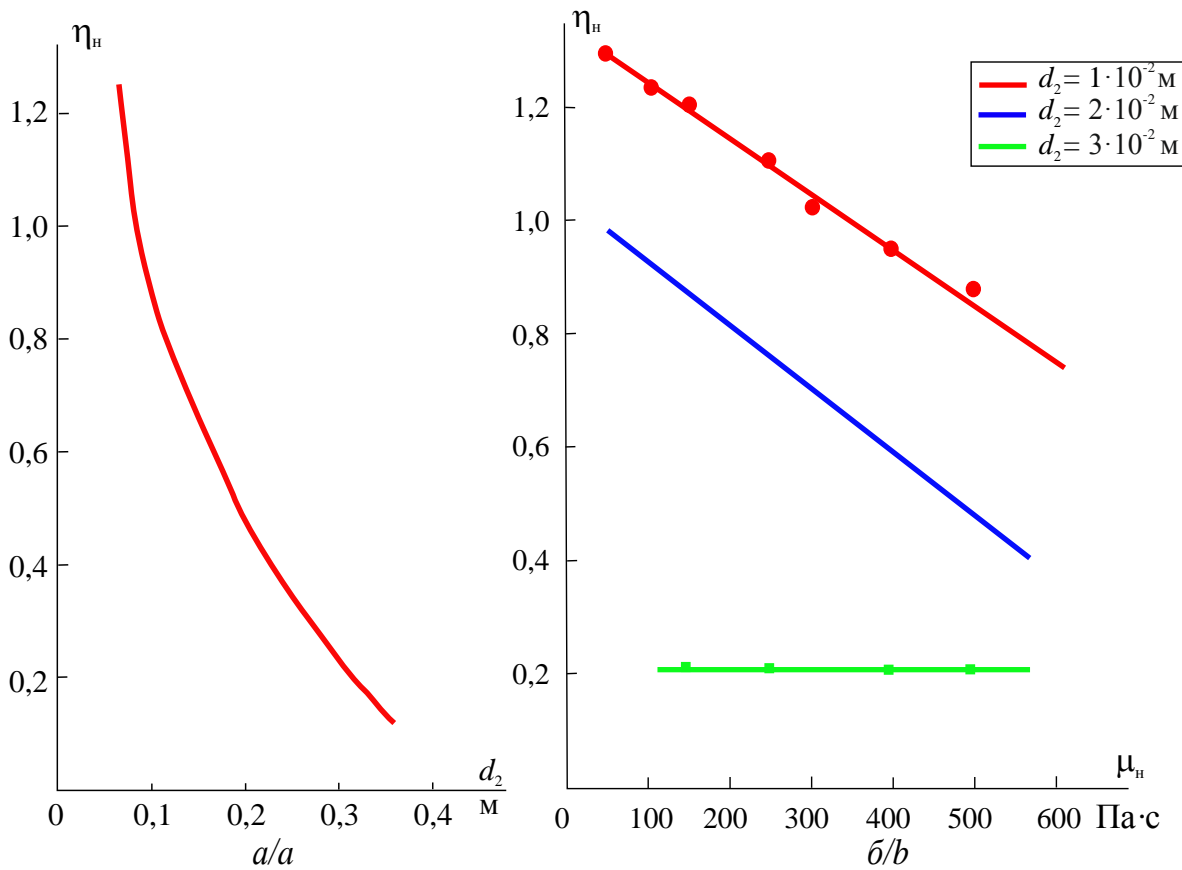

Pис. 5. Зависимость изменения $\eta_{н}$ в нефти от изменения: а) толщины слоя при динамической вязкости $\mu_{н}=0,5$ Па.с и при частоте воздействия 160 кГи; б) вязкости нефти при частоте воздействия 160 кГи и толшине слоя нефти $d_{2}=1 \cdot 10^{-2} \mathrm{M} ; 2 \cdot 10^{-2} \mathrm{M} ; 3 \cdot 10^{-2} \mathrm{M}$

Fig. 5. Dependence of coefficient $\eta_{n}$ change in oil on the changes in: a) layer thickness at dynamic viscosity $\mu_{n}=0,5$ Pa:sec and with the frequency of exposure $160 \mathrm{kHz} ; \mathrm{b}$ ) oil viscosity at the frequency of exposure $160 \mathrm{kHz}$ and the thickness of the oil layer $d_{2}=1 \cdot 10^{-2} \mathrm{~m} ; 2 \cdot 10^{-} \mathrm{m} ; 3 \cdot 10^{-2} \mathrm{~m}$

В дальнейших исследованиях планируется расширение возможностей представленной математической модели с целью определения степени нагрева среды, изменения реологических свойств конкретного объема нефти в стационарном и переходном режимах работы акустической системы.

\section{Выводы}

Разработанная математическая модель распространения ультразвука в многослойной среде позволяет рассчитать энергию в каждом слое акустической системы при различных частотах возбуждения источника колебаний с различными соотношениями длины волны в слое и толщины данного слоя.

Математическая модель учитывает коэффициенты поглощения в слоях акустической системы и отраженные волны в каждом слое. Наличие отраженных волн в каждом слое создает череду режимов стоячих

\section{СПИСОК ЛИТЕРАТУРЬ}

1. Лоскутова Ю.В., Юдина Н.В., Рикконен С.В. Результаты физико-химической обработки высокозастывающих нефтей // Химия нефти и газа: Международная конференция. - Томск, 2018. - C. 633-634.

2. Intensification of the processes of preparation of drilling and cement mortars using vibration jet activation methods S.V. Rikkonen, M.A. Dmitrieva, V.N. Leitsin, S.V. Ponomarev, A.V. Azin // IOP Conf. Ser: Mater. Sci. Eng. - 2020 - V. 911. № 012001. DOI: 10.1088/1757-899X/911/1/012001. волн и, как следствие, множество максимумов и минимумов на частотной характеристике слоев.

Частотные характеристики с учетом интерференционной картины поля отражают картину проникновения энергии в слои и показывают, что за счет образования стоячих волн и накопления энергии в слое отношение $\eta_{\mathrm{n}}$ данного слоя к входящей энергии (при малых поглощениях в слоях) может быть близко к 1 или даже больше 1 , что согласуется с источниками $[18,19]$

Акустическая энергия в слое нефти падает в зависимости от увеличения исходной вязкости.

Представленная математическая модель позволяет рассчитать конструкцию акустической системы для подготовки нефти и углеводородных топлив к транспорту, подготовки топлив к сжиганию, подготовки масел к эксплуатации в условиях Арктики и Антарктики.

3. Способ виброструйной гидродинамической технологии сохранения текучести углеводородных топлив и нефтепродуктов в условиях низких температур / А.В. Азин, Е.П. Богданов, С.В. Пономарев, С.В. Рикконен // Известия Томского политехнического университета. Инжиниринг георесурсов. 2019. - Т. 330. - № 4. - С. 158-177.

4. Идентификация параметров механической системы вибрационного электромагнитного активатора по граничным околорезонансным частотам / А.Н. Гаврилин, С.Н. Кладиев, А.С. Глазырин, Е.В. Боловин, В.И. Полищук // Известия Томского по- 
литехнического университета. Инжиниринг георесурсов. 2019. - Т. 330. - № 4. - С. 158-177.

5. Резонансные колебания с предельной амплитудой в вибрационном электромагнитном активаторе / А.Н. Гаврилин, Е.В. Боловин, А.С. Глазырин, С.Н. Кладиев, В.И. Полищук // Известия Томского политехнического университета. Инжиниринг георесурсов. - 2019. - Т. 330. - № 1. - С. 201-213.

6. Создание эталонной плоской ультразвуковой волны в жидкости с помощью плоского пьезоэлектрического преобразователя большого волнового размера / А.А. Крохмаль, Д.А. Николаев, С.А. Цысарь, О.А. Сапожников // Акустический журнал. - 2020. - Т. 66. - № 5. - С. 475-488.

7. Исаев А.Е., Айвазян Ю.М., Поликарпов А.М. Проблемы исследования акустических свойств материалов методами ближнего поля // Альманах современной метрологии. 2020. - № 1. - С. 163-196.

8. Зайцев В.В., Кузнецова И.Е. Акустические волны в тонких пьезоэлектрических пластинах. - М.: Радиотехника, 2018. $240 \mathrm{c}$.

9. Thin film flexible/bendable acoustic wave devices: Evolution, hybridization and decoupling of multiple acoustic wave modes / R. Tao, W.B. Wang, J.T. Luo, S.A. Hasan, H. Torun, P. CanyellesPericas, J. Zhou, W.P. Xuan, M.D. Cooke, D. Gibson, Q. Wu, W.P. Ng, J.K. Luo, Y.Q. Fu // Surface \& Coatings Technology. 2019. - V. 357. - P. 587-594.

10. Guiding and splitting Lamb waves in coupled-resonator elastic waveguides / Y.-F. Wang, T.-T. Wang, J.-P. Liu, Y.-S. Wang, V. Laude // Composite Structures. - 2018. - V. 206. - P. 588-593.

11. Анисимкин В.И., Воронова Н.В. Особенности генерации нормальных акустических волн высших порядков в тонких пьезо- электрических пластинах // Акустический журнал. - 2020. Т. 66. - № 1. - С. 3-7.

12. Гусев В.А., Руденко О.В. Поля радиационных сил и акустические течения в жидком слое на твердом полупространстве // Акустический журнал. - 2019. - Т. 65. - № 2. - С. 166-181.

13. Guz A.N., Bagno A.M. Effect of prestresses on the dispersion of Lamb waves in a system consisting of a viscous liquid layer and a compressible elastic layer // International applied mechanics.2018. - V. 54 (3). - P. 249-258.

14. Huang L., Liang J., Wu C. A three-dimensional indirect boundary integral equation method for modeling elastic wave scattering in a layered half-space // Int. J. Solids Structures. - 2019. - V. 169. P. 81-94.

15. Falleta S., Monegato G., Scuderi L. On the discretization and application of two space-time boundary integral equations for $3 \mathrm{D}$ wave propagation problems in unbounded domains // Applied Numerical Mathematics. - 2018. - V. 124. - P. 22-43.

16. Research on the transmission characteristics of air-coupled ultrasound in double-layered bonded structures / X.-G. Wang, W.-L. Wu, Z.-C. Huang, J.-J. Chang, N.-X. Wu // Materials. 2018. - V. 11. - № 310. DOI: 10.3390/ma11020310.

17. Хаясака Т. Электроакустика. - М.: Мир, 1982. - 248 с.

18. Xu Y., Langbauer C., Hofstaetter $\mathrm{H}$. The application of ultrasonic technology for cleaning oil contaminated sand // SPE Asia Pacific Health, Safety, Security, Environment and Social Responsibility Conference. - Kuala Lumpur, 2017. - 12 p. DOI: 10.2118/185261-ms.

19. Исакович М.А. Общая акустика. - М.: Наука, 1973. - 489 с.

20. Соколов В.С. Дефектоскопия материалов. - М.: Госэнергоиздат, 1961. $-328 \mathrm{c}$

Поступила 07.04.2021 2.

\section{Информация об авторах}

Aзин A.B., кандидат физико-математических наук, старший научный сотрудник Научно-исследовательского института прикладной математики и механики Национального исследовательского Томского государственного университета.

Богданов Е.П., кандидат технических наук, доцент Инженерной школы энергетики Национального исследовательского Томского политехнического университета.

Марицкий H.H., кандидат физико-математических наук, инженер-исследователь Научно-исследовательского института прикладной математики и механики Национального исследовательского Томского государственного университета.

Пономарев С.A., аспирант, инженер-исследователь Научно-исследовательского института прикладной математики и механики Национального исследовательского Томского государственного университета.

Рикконен C.B., кандидат технических наук, доцент, инженер-исследователь Научно-исследовательского института прикладной математики и механики Национального исследовательского Томского государственного университета. 
UDC 534.21

\title{
MATHEMATICAL MODELLING OF HIGH-VISCOUS OIL ULTRASONIC PREPARATION FOR TRANSPORT
}

\author{
Anton V. Azin'1, \\ antonazin@niipmm.tsu.ru
}

Eugene P. Bogdanov², epbogdanov@mail.ru

Nikolay N. Maritsky ${ }^{1}$, nnmar@niipmm.tsu.ru

Sergey A. Ponomarev ${ }^{1}$, doc.sergeyponomarev@gmail.com

\author{
Sergey V. Rikkonen', \\ rikk2@yandex.ru \\ 1 National Research Tomsk State University, \\ 36, Lenin avenue, Tomsk, 634050, Russia. \\ 2 National Research Tomsk Polytechnic University, \\ 30, Lenin avenue, Tomsk, 634050, Russia.
}

The relevance of the research is caused by the need to determine the energy level of physical effects on high-viscous oil in order to prepare it for transport.

This knowledge is relevant for the design of ultrasonic installations for changing the rheological properties of oil.

The main aim of the research is to determine acoustic energy levels in layers of multilayer acoustic system at different impact frequencies in order to identify resonant modes of the mechanical system «emitter-oil».

Objects: mechanical multi-layered system «emitter-oil», the design of which is determined by the method of energy application to the load and the resonant frequency of the particular layer.

Methods: mathematical modeling of ultrasonic radiation with the determination of the energy of transmission, absorption and reflection in each layer of the mechanical system «emitter-oil», determination of resonant frequencies with the maximum released energy in the oil layer.

Results. Mathematical model of a multilayer acoustic system is presented. It allows calculating the energy of acoustic radiation in each layer of the system. The frequency characteristics of acoustic radiation make it possible to determine the resonance modes in each layer of the system, including oil. Knowledge of the level of acoustic energy, in the future, will make it possible to determine changes in the rheological properties of oil, including from heating.

Findings. The developed mathematical model of the ultrasound propagation in a multilayer system makes it possible to calculate the energy in each layer of the acoustic system at different excitation frequencies of the vibration source with different ratios of the wavelength in the layer and the thickness of this layer. The presence of reflected waves in each layer creates a series of resonances (standing wave modes). The frequency characteristics, taking into account the interference pattern of the field, reflect the pattern of energy penetration into the layers and show that due to the formation of standing waves (resonances), the energy in oil can be close to the energy of the emitter. The acoustic energy in the oil layer decreases depending on the increase of the initial viscosity. This mathematical model makes it possible to calculate the design of an acoustic system for preparing oil and hydrocarbon fuels for transport, preparing fuels for combustion, preparing oils for operation in the Arctic and Antarctic.

\section{Key words:}

Ultrasonic radiation, radiation energy, standing waves, reflected waves, frequency characteristics, layered acoustic system.

\section{REFERENCES}

1. Loskutova Yu.V., Yudina N.V., Rikkonen S.V. Rezultaty fizikokhimicheskoy obrabotki vysokozastyvayushchikh neftey [Results of physicochemical treatment of highly solidifying oils]. Mezhdunarodnaya konferentdsiya. Khimiya nefti i gaza [International Conference. Chemistry of Oil and Gas]. Tomsk, 2018. pp. 633-634.

2. Rikkonen S.V., Dmitrieva M.A., Leitsin V.N., Ponomarev S.V., Azin A.V. Intensification of the processes of preparation of drilling and cement mortars using vibration jet activation methods. IOP Conf. Ser.: Mater. Sci. Eng, 2020, vol. 911, no. 012001. DOI: 10.1088/1757-899X/911/1/012001.

3. Azin A.V., Bogdanov E.P., Ponomarev S.V., Rikkonen S.V Method of vibro-jet hydrodynamic technology to retain the fluidity of hydrocarbon fuels and petroleum products at low temperatures. Bulletin of the Tomsk Polytechnic University. Geo Assets Engineering, 2019, vol. 330, no. 4, pp. 41-48. In Rus.
4. Gavrilin A.N., Kladiev S.N., Glazyrin A.S., Bolovin E.V., Polishchuk V.I. Identification of parameters of vibration electromagnetic activator mechanical system using limiting near resonance frequency. Bulletin of the Tomsk Polytechnic University. Geo Assets Engineering, 2019, vol. 330, no. 4, pp. 158-177. In Rus.

5. Gavrilin A.N., Bolovin E.V., Glazyrin A.S., Kladiev S.N., Polishchuk V.I. Resonant oscillations with a limiting amplitude in a vibration electromagnetic activator. Bulletin of the Tomsk Polytechnic University. Geo Assets Engineering, 2019, vol. 330, no. 1, pp. 201-213. In Rus.

6. Krohmal A.A., Nikolaev D.A., Tsysar S.A., Sapozhnikov O.A. Sozdanie etalonnoy ploskoy ultrazvukovoy volny $\mathrm{v}$ zhidkosti $\mathrm{s}$ pomoshchyu ploskogo pyezoelektricheskogo preobrazovatelya bolshogo volnovogo razmera [Creation of a reference plane ultrasonic wave in a liquid using a flat piezoelectric transducer of large wave size]. Akusticheskiy zhurnal, 2020, vol. 66, no. 5, pp. 475-488. 
7. Isaev A.E., Aivazian Yu.M., Polikarpov A.M. Problems of research of acoustic properties of materials by methods of near field. Almanac of modern metrology, 2020, no. 1, pp. 163-196. In Rus.

8. Zaytsev V.V., Kuznetsova I.E. Akusticheskie volny v tonkikh pyezoelektricheskikh plastinakh [Acoustic waves in thin piezoelectric plates]. Moscow, Radiotehnika Publ., 2018. 240 p.

9. Tao R., Wang W.B., Luo J.T., Hasan S.A., Torun H., CanyellesPericas P., Zhou J., Xuan W.P., Cooke M.D., Gibson D., Wu Q., Ng W.P., Luo J.K., Fu Y.Q. Thin film flexible/bendable acoustic wave devices: Evolution, hybridization and decoupling of multiple acoustic wave modes. Surface \& Coatings Technology, 2019, vol. 357, pp. 587-594.

10. Wang Y.-F., Wang T.-T., Liu J.-P., Wang Y.-S., Laude V. Guiding and splitting Lamb waves in coupled-resonator elastic waveguides. Composite Structures, 2018, vol. 206, pp. 588-593.

11. Anisimkin V.I., Voronova N.V. Osobennosti generatsii normalnykh akusticheskikh voln vysshikh poryadkov tonkikh pyezojelektricheskikh plastinakh [Features of the generation of higher-order normal acoustic waves in thin piezoelectric plates]. Akusticheskiy zhurnal, 2020, vol. 66, no. 1, pp. 3-7.

12. Gusev V.A., Rudenko O.V. Polya radiatsionnykh sil i akusticheskie techeniya $\mathrm{v}$ zhidkom sloe na tverdom poluprostranstve [Radiation force fields and acoustic flows in a liquid layer on a solid halfspace]. Akusticheskiy zhurnal, 2019, vol. 65, no. 2, pp. 166-181.

13. Guz A.N., Bagno A.M. Effect of prestresses on the dispersion of Lamb waves in a system consisting of a viscous liquid layer and a compressible elastic layer. International applied mechanics, 2018, vol. 54 (3), pp. 249-258.

14. Huang L., Liang J., Wu C. A three-dimensional indirect boundary integral equation method for modeling elastic wave scattering in a layered half-space. Int. J. Solids Structures, 2019, vol. 169, pp. 81-94.

15. Falleta S., Monegato G., Scuderi L. On the discretization and application of two space-time boundary integral equations for 3D wave propagation problems in unbounded domains. Applied $\mathrm{Nu}$ merical Mathematics, 2018, vol. 124, pp. 22-43.

16. Wang X.-G., Wu W.-L., Huang Z.-C., Chang J.-J., Wu N.-X. Research on the transmission characteristics of air-coupled ultrasound in double-layered bonded structures. Materials, 2018, vol. 11, no. 310. DOI: $10.3390 /$ ma11020310.

17. Hayasaka T. Elektroakustika [Electroacoustics]. Moscow, Mir Publ., 1982. $248 \mathrm{p}$

18. Xu Y., Langbauer C., Hofstaetter H. The application of ultrasonic technology for cleaning oil contaminated sand. SPE Asia Pacific Health, Safety, Security, Environment and Social Responsibility Conference. Kuala Lumpur, 2017. 12 p. DOI: 10.2118/185261-ms.

19. Isakovich M.A. Obshchaya akustika [General acoustics]. Moscow, Nauka Publ., 1973. 489 p.

20. Sokolov V.S. Defektoskopiya materialov [Flaw detection of materials]. Moscow, Gosjenergoizdat Publ., 1968. 328 p.

Received: 7 April 2021.

\section{Information about the authors}

Anton V. Azin, Cand. Sc., senior research officer, National Research Tomsk State University.

Eugene P. Bogdanov, Cand. Sc., associate professor, National Research Tomsk Polytechnic University.

Nikolay N. Maritsky, Cand. Sc., research engineer, National Research Tomsk State University.

Sergey A. Ponomarev, research engineer, postgraduate, National Research Tomsk State University.

Sergey V. Rikkonen, Cand. Sc., associate professor, research engineer, National Research Tomsk State University. 